\title{
Soluble Host-Defense Lectins
}

\author{
Misao Matsushita, ${ }^{1}$ David C. Kilpatrick, ${ }^{2}$ Bok Luel Lee, ${ }^{3}$ and Nobutaka Wakamiya ${ }^{4}$ \\ ${ }^{1}$ Department of Applied Biochemistry, Tokai University, Hiratsuka 259-1292, Japan \\ ${ }^{2}$ Scottish National Blood Transfusion Service, National Science Laboratory, Edinburgh EH177QT, UK \\ ${ }^{3}$ National Research Laboratory of Defense Proteins, Global Research Laboratory of Insect Symbiosis, College of Pharmacy, \\ Pusan National University, Busan 609735, Republic of Korea \\ ${ }^{4}$ Department of Microbiology and Immunology, Asahikawa Medical University, Asahikawa 078-8510, Japan
}

Correspondence should be addressed to Misao Matsushita, mmatsu@keyaki.cc.u-tokai.ac.jp

Received 3 July 2012; Accepted 3 July 2012

Copyright (C) 2012 Misao Matsushita et al. This is an open access article distributed under the Creative Commons Attribution License, which permits unrestricted use, distribution, and reproduction in any medium, provided the original work is properly cited.

Lectins are proteins that bind to carbohydrates and are distributed widely in animals, plants, bacteria, and so forth. Animal lectins have a role in many biological functions including development and immunity. There are two kinds of immune system in vertebrates: innate immunity and adaptive (or acquired) immunity. Innate immunity plays an important role at an early stage of infection, when phagocytic cells (neutrophils and macrophages), NK cells, and complement are major components. Adaptive immunity takes over from the innate immune system as time passes. In adaptive immunity, antibodies, $\mathrm{T}$ cells, and $\mathrm{B}$ cells are major players. One of the striking differences between innate immunity and adaptive immunity is in recognition specificity for pathogens. Antibodies and T cells are able to recognize pathogens or their derived peptides with high specificity. On the other hand, the recognition specificity of innate immunity is broad. Invertebrates have innate immunity but not adaptive immunity.

Proteins known as pattern-recognition proteins recognize components peculiar to microbes on their surfaces. These components are called pathogen-associated molecular patterns (PAMPs), including lipopolysaccharide, peptidoglycan, lipoteichoic acid, and $\beta$-glucan. Many soluble animal lectins involved as pattern-recognition proteins have been identified, and their structures and functions have been characterized. These lectins are termed "soluble hostdefense lectins" that include collectins, ficolins, galectins, and pentraxins. Soluble host-defense lectins comprise several groups of proteins defined by their structural features. For instance, collectins are proteins that consist of a collagenlike domain and a carbohydrate-recognition domain and include mannose-binding lectin (MBL), SP-A, SP-D, and CLK1. Ficolins consist of a collagen-like domain combined with a fibrinogen-like domain and include human L-ficolin and mouse ficolin A. Soluble host-defense lectins bind to PAMPs of pathogens as a recognition molecule and elicit immune effector mechanisms including enhancement of phagocytosis and activation of the complement system. Enhancement of phagocytosis involves specific receptors for soluble hostdefense lectins present on the membranes of phagocytes.

The complement system is an effector system in immunity. It consists of many proteins involved in a cascade of proteolysis and protein complex assembly that leads to the elimination of pathogens in several ways including enhancement of phagocytosis and direct killing. The system is activated via three pathways (the classical, alternative, and lectin pathways). The lectin pathway is an important component of innate immunity and is activated by the binding of serum ficolins or MBL to carbohydrates on the pathogen surface. These soluble host-defense lectins form complexes with three types of MBL-associated serine proteases (MASPs) and their truncated proteins. It was shown that CL-K1 is also complexed with MASPs. In the lectin pathway, the binding of the ficolin-MASP complex or the MBL-MASP complex (i.e., a complex composed of ficolins or MBL, MASPs, and their truncated proteins) to carbohydrates on the microbe surface initiates the activation of complement, leading to the elimination of microbes.

This special issue, including 4 original articles and 6 review articles, focuses on "soluble host-defense lectins" in terms of their structure, function, and physiological and pathological relevance. Many of the papers deal with 
collectins: the functions of novel collectins including CL$\mathrm{K} 1$ (K. Ohtani et al.), the role of MBL in inflammation (M. Larvie et al.), pathological relevance of MBL in IgA nephrology (I. Ohsawa et al.), anti-influenza virus activity of collectins (W. C. Ng et al.), and diverse functions of pulmonary collectins (S. Ariki et al.). Ficolins are also discussed in two papers regarding the clinical significance of L-ficolin (D. C. Kilpatrick and J. D. Chalmers) and the ability of mouse ficolin B to activate the lectin complement pathway (Y. Endo et al.). M. Cedzynski et al. report a role for MBL and ficolins in the protection against infection in neonates. A paper concerning the role of DC-SIGN, a lectin on dendritic cells, in bacterial interaction (E. Miszczyk et al.) and a paper describing the association between the genetic polymorphisms of CLEC5A, a lectin on myeloid cells, and Kawasaki disease (Y.-L. Yang et al.) are also included in this issue.

Misao Matsushita David C. Kilpatrick Bok Luel Lee

Nobutaka Wakamiya 

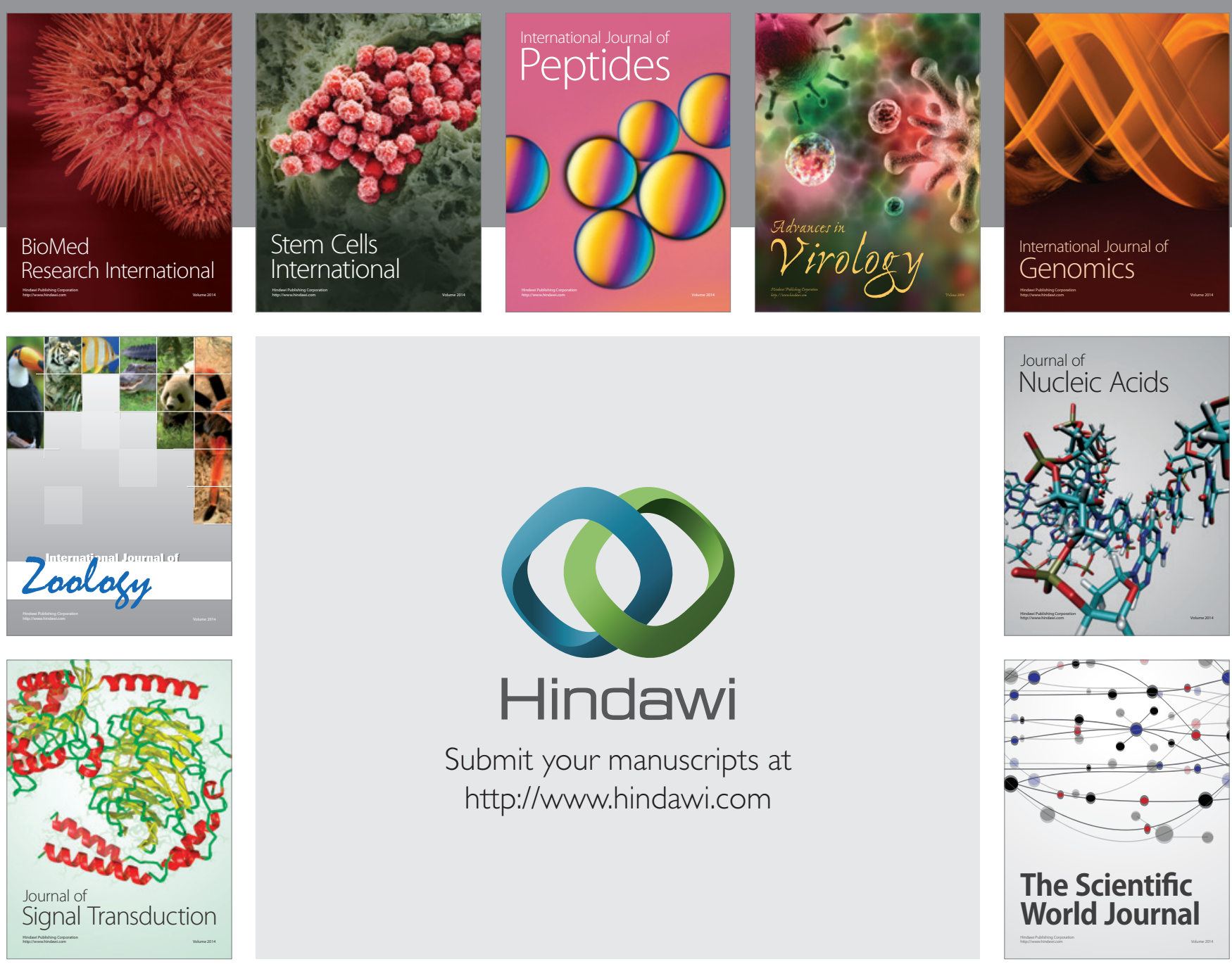

Submit your manuscripts at

http://www.hindawi.com
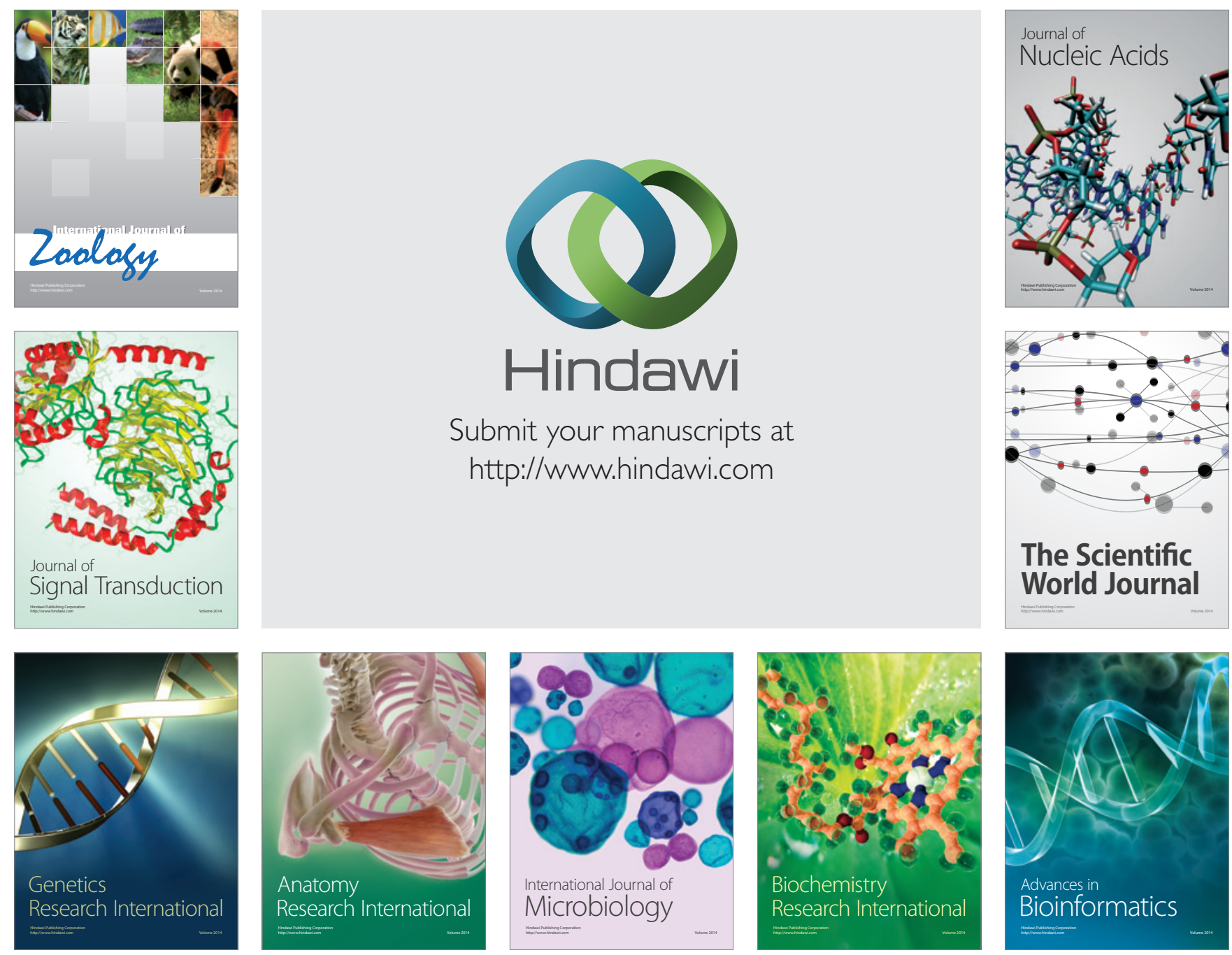

The Scientific World Journal
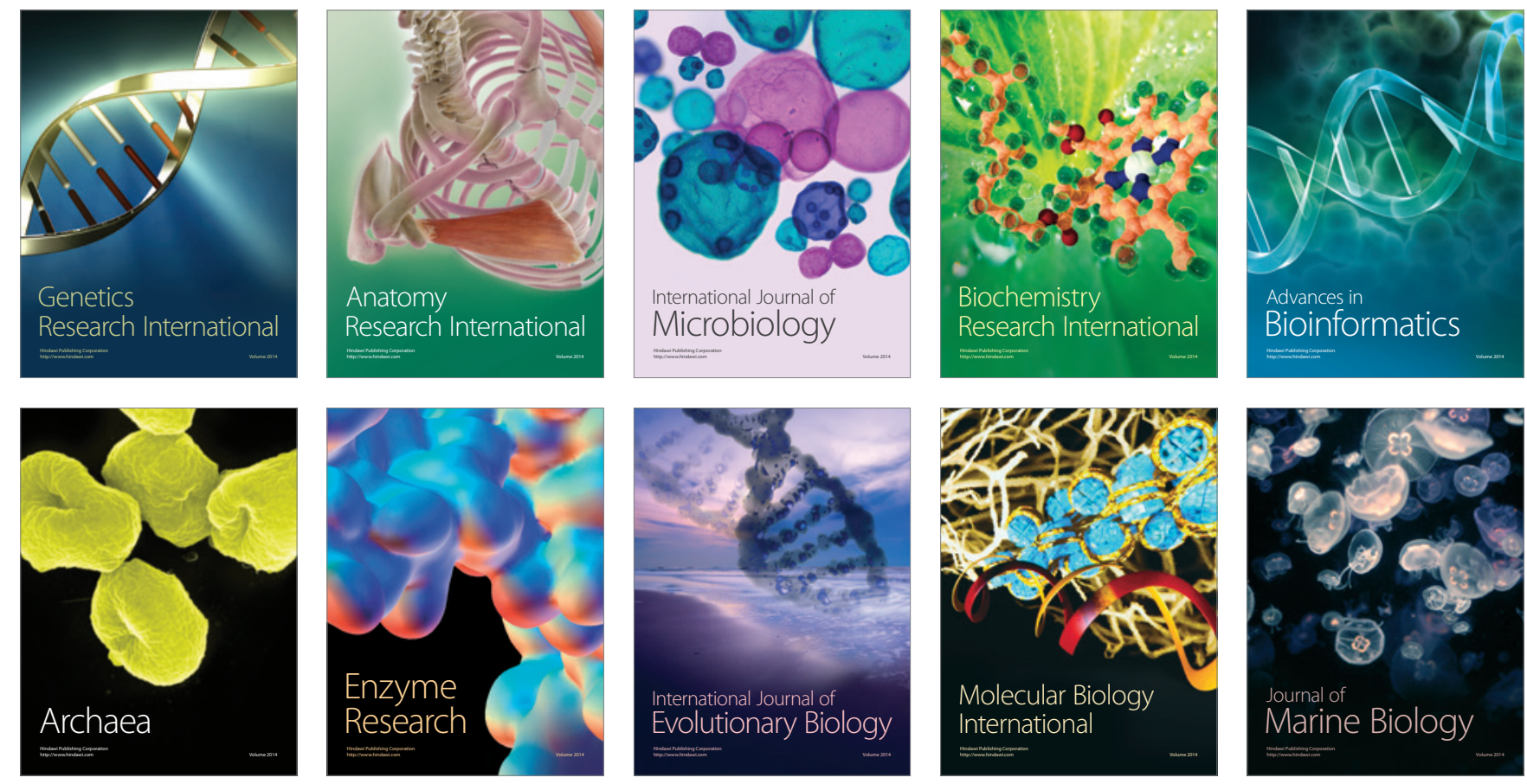